\title{
Evaluating a multi-component, community- based program to improve adherence and retention in care among adolescents living with HIV in Zimbabwe: study protocol for a cluster randomized controlled trial
}

\author{
Webster Mavhu ${ }^{1,2^{*}}$ (D) Nicola Willis ${ }^{3}$, Juliet Mufuka', Collin Mangenah¹, Kudzanayi Mvududu', Sarah Bernays ${ }^{4}$, \\ Walter Mangezi ${ }^{5}$, Tsitsi Apollo ${ }^{6}$, Ricardo Araya ${ }^{7}$, Helen A. Weiss ${ }^{8}$ and Frances M. Cowan ${ }^{1,2}$
}

\begin{abstract}
Background: World Health Organization (WHO) adolescent HIV-testing and treatment guidelines recommend community-based interventions to support antiretroviral therapy (ART) adherence and retention in care, while acknowledging that the evidence to support this recommendation is weak. This cluster randomized controlled trial aims to evaluate the effectiveness and cost-effectiveness of a psychosocial, community-based intervention on HIVrelated and psychosocial outcomes.

Methods/design: We are conducting the trial in two districts. Sixteen clinics were randomized to either enhanced ART-adherence support or standard of care. Eligible individuals (HIV-positive adolescents aged 13-19 years and eligible for ART) in both arms receive ART and adherence support provided by adult counselors and nursing staff. Adolescents in the intervention arm additionally attend a monthly support group, are allocated to a designated community adolescent treatment supporter, and followed up through a short message service (SMS) and calls plus home visits. The type and frequency of contact is determined by whether the adolescent is "stable" or in need of enhanced support. Stable adolescents receive a monthly home visit plus a weekly, individualized SMS. An additional home visit is conducted if participants miss a scheduled clinic appointment or support-group meeting. Participants in need of further, enhanced, support receive bi-weekly home visits, weekly phone calls and daily SMS. Caregivers of adolescents in the intervention arm attend a caregiver support group. Trial outcomes are assessed through a clinical, behavioral and psychological assessment conducted at baseline and after 48 and 96 weeks. The primary outcome is the proportion who have died or have virological failure (viral load $\geq 1000$ copies $/ \mathrm{ml}$ ) at 96 weeks. Secondary outcomes include virological failure at 48 weeks, retention in care (proportion of missed visits) and psychosocial outcomes at both time points. Statistical analyses will be conducted and reported in line with CONSORT guidelines for cluster randomized trials, including a flowchart.

(Continued on next page)
\end{abstract}

\footnotetext{
* Correspondence: wmavhu@gmail.com

${ }^{1}$ Centre for Sexual Health and HIV/AIDS Research (CeSHHAR), 9 Monmouth

Road, Avondale West, Harare, Zimbabwe

${ }^{2}$ Department of International Public Health, Liverpool School of Tropical

Medicine, Liverpool, UK

Full list of author information is available at the end of the article
} 
(Continued from previous page)

Discussion: This study provides a unique opportunity to generate evidence of the impact of the on-going Zvandiri program, for adolescents living with HIV, on virological failure and psychosocial outcomes as delivered in a realworld setting. If found to reduce rates of treatment failure, this would strengthen support for further scale-up across Zimbabwe and likely the region more widely.

Trial registration: Pan African Clinical Trial Registry database, registration number PACTR201609001767322 (the Zvandiri trial). Retrospectively registered on 5 September 2016.

Keywords: HIV, Antiretroviral therapy, Adolescents, Adherence, Zimbabwe, Psychosocial, Community-based

\section{Background}

In sub-Saharan Africa, the number of HIV-related deaths among adolescents (aged 10-19 years) tripled between 2000 and 2014 despite decreasing among all other age groups. HIV is now the leading cause of death among this age group $[1,2]$. The increase in adolescent HIVrelated deaths has been attributed to poor prioritization of adolescents in national HIV plans, inadequate provision of accessible and acceptable HIV-testing, counseling and treatment services for this age group, plus a lack of tailored support for adolescents to remain in care and adhere to taking antiretroviral therapy (ART) [3-5]. Adolescents in sub-Saharan Africa have poorer ART adherence than adults [6] and are at high risk of loss to follow-up from treatment and care [7]. This supports findings among adolescents with other chronic childhood illnesses (e.g., diabetes, asthma) when medication adherence frequently becomes worse as the child becomes more independent from parental control as they enter adolescence [8].

In 2013, the World Health Organization (WHO) released the first consolidated ART prevention and treatment guidelines which recommended innovative models of ART delivery (decentralization, integration and taskshifting) [9]. In response, Zimbabwe revised its national ART guidance, released in December 2013, to simplify recommended treatment regimens for children and adolescents, hence facilitating the decentralization of pediatric HIV care to primary healthcare facilities [10]. Decentralizing pediatric HIV care has resulted in improved access and coverage, but little is known about the capacity of primary care facilities to provide effective adherence support as well as support for the broader psychosocial issues that many of these children and adolescents face [4].

A key tenet of both the WHO and Zimbabwe's treatment guidelines is that expanded access to ART needs to be accompanied by interventions to maximize retention in care and medication adherence. Poor retention and adherence to ART leads to treatment resistance and ultimately, to treatment failure $[5,11,12]$. International guidelines recommend at least $95 \%$ adherence to ART to minimize the risk of virological failure [13-15]. A systematic review of 17 studies relating to ART programs in children and adolescents from low- and middleincome countries (LMIC) found that non-adherence was associated with a child's socioeconomic environment, stigma, caregiver characteristics, dynamics of childcaregiver interactions, disclosure of HIV status, regimen complexity, side effects, and cost of medication [16].

The 2013 and 2015 WHO adolescent HIV-testing and treatment guidelines recommend community-based interventions to support ART adherence and retention in care, while acknowledging that the evidence to support this recommendation is weak [4, 17]. Evidence-based strategies to provide support to the growing number of adolescents receiving ART from LMIC are urgently required. Preliminary evidence suggests that the Zvandiri ("As I am") program (http://www.africaid-zvandiri.org/) has the potential to make a significant impact on the lives of adolescents living with HIV [18].

The Zvandiri program is a model of differentiated clinical service delivery for HIV-positive children and adolescents in Zimbabwe, has been cited as a "best practice" intervention by the WHO [4], USAID, Southern African Development Community [19], and has been recommended for adaptation and regional scale-up [19]. The program uses trained and mentored community adolescent treatment supporters (CATS) to deliver structured support groups and tailored community-based adherence support coupled with counseling for the broader psychosocial issues that these young people are facing (including coming to terms with their emerging sexuality in the context of a life-threatening, sexually transmissible infection). The CATS are aged 18-23 years, living with HIV, have been identified by the healthcare facility as committed, competent and motivated to support their peers as well as adherent to ART, having completed secondary schooling and having consent from their caregivers to enroll as CATS. The Zvandiri program also includes an intervention for caregivers which focuses on enhancing their knowledge, skills and confidence of HIV and treatment literacy as well as communication and parenting through a participatory training program. 
In this paper we describe the protocol for a cluster randomized controlled trial (cRCT) to evaluate the effectiveness and cost-effectiveness of the Zvandiri program on HIV-related and psychosocial outcomes.

\section{Methods/design \\ Objectives}

The trial will provide evidence on whether enhancing community-based support for adolescents on ART through the Zvandiri program will, compared with standard care:

1. Reduce the proportion of adolescents with ART treatment failure (lack of viral suppression)

2. Improve retention in care

3. Reduce the severity of anxiety and depression

4. Reduce the proportion of adolescents reporting nondisclosure

5. Be cost-effective

\section{Hypotheses}

The primary hypothesis is that the Zvandiri program will be more effective than standard care in reducing the proportion of adolescents who have died or who are failing treatment at 96 weeks (defined as a viral load $\geq 1000$ copies/ml).

The secondary hypotheses are that the Zvandiri program (1) improves virological failure at 48 weeks, (2) improves retention in care, (3) reduces psychological distress, (4) increases HIV status disclosure to sexual partners and (5) decreases perceived stigma.

\section{Trial setting}

The trial is being conducted in two districts (Bindura and Shamva) in Mashonaland Central Province. The districts were selected in consultation with the Ministry of Health and Child Care (MoHCC). At the time of selection, it was estimated that 24,871 adolescents were living with HIV in the province and that ART coverage among children and adolescents was the lowest in Zimbabwe (estimated to be 29\%). Additionally, although the Zvandiri program was being scaled-up, it had not yet been initiated in these two districts. Altogether, there are 33 healthcare facilities in the two districts. Of these, 16 with at least 20 adolescents on ART (excluding private clinics and those not yet offering pediatric HIV treatment and care services) were selected to participate.

\section{Trial design}

The trial is a cluster randomized controlled trial (cRCT). The clinic is the unit of randomization. Sixteen clinics were randomized to either enhanced ART-adherence support through the Zvandiri program or standard of HIV care in a 1:1 allocation (Fig. 1). Adolescents aged 13-19 years were recruited. Trial outcomes are assessed through a clinical, behavioral and psychological assessment conducted at baseline and after 48 and 96 weeks.

\section{Eligibility criteria}

1. Inclusion criteria: HIV-positive adolescents, aged 13-19 years, eligible for ART (i.e., either starting or already on ART) and able to provide informed assent and their caregiver is able to provide 
informed consent (those aged 18 and 19 years do not need caregiver consent)

2. Exclusion criteria: too physically or psychologically unwell or unable to give informed consent

\section{Interventions}

\section{Control arm}

All eligible adolescents attending clinics allocated to the standard-care arm receive ART and adherence support as set out in the prevailing MoHCC guidelines [20]. As per MoHCC guidelines, adherence support is provided by adult counselors and nursing staff. After ART initiation, adolescents are seen monthly, with cluster of differentiation 4 (CD4) monitoring at 6-monthly intervals. Prescription refills, pill counts and participant interviews are used to measure adherence. Although routine viralload testing was adopted by MoHCC in 2010 and scaleup began in 2012 [21], the MoHCC is currently only able to provide targeted rather than universal viral-load testing.

\section{Intervention arm}

All eligible adolescents attending intervention clinics will receive $\mathrm{MoHCC}$ standard care. In addition, one to three (ideally at least one man, one woman) trained and supported CATS are at each clinic to provide adherence counseling and support to adolescents at their clinic visits as well as on-going individualized communitybased support. Each CATS supports 6-10 adolescents in their geographical location irrespective of level of Zvandiri support. CATS were recruited from the clinic in partnership with the clinic staff, or from the local support group if a group was already in place.

The CATS receive 2 weeks of MoHCC-endorsed training to equip them with counseling skills and skills in community outreach plus knowledge specific to adolescent HIV (ART and adherence, disclosure, sexual and reproductive health, psychosocial support and mental health). The training combines both theoretical and practical components, including the "shadowing" of existing CATS in Harare to acquire practical experience of providing adherence support. Training includes learning how to identify "red flags" for ill-health, adherence challenges, psychosocial distress and common mental disorders (CMDs), plus basic skills in problem solving and cognitive behavioral therapy (CBT). Training is participatory and uses case studies. All CATS attend a weekly supervision meeting at the clinic with a designated nurse and provide each other with peer-to-peer support (through a WhatsApp group and via Skype from Harare) with oversight from a professional counselor. Trial participants are allocated to a designated CATS according to their residential area and followed up through the short message service (SMS), phone calls and home visits. The type and frequency of contact is determined following assessment of their individual situation (i.e., whether they are considered "stable" or in need of enhanced support):

1. "Stable" adolescents are those: (a) with a viral load < 1000 copies/ml and/or most recent (and within the last 6 months) CD4 count $\geq 200$ cells $/ \mathrm{ml}$ and (b) attending all scheduled clinic visits in the last 3 months assessed using the patient-held record. Stable adolescents receive a home visit once a month, plus a weekly, individualized SMS. The SMS is written and sent by the CATS and focuses on (i) motivational reminders around adherence, (ii) clinic and support group attendance and (iii) encouragement to contact the CATS in the event of any problems. An additional home visit is conducted if an adolescent misses a scheduled clinic appointment or support-group meeting

2. Adolescents in need of enhanced support are those: (a) with a viral load $\geq 1000$ copies/ml and/or CD4 count $<200$ cells/ml, (b) at risk of other CMDs/with a major depressive disorder (assessment methods described below), (c) failing to attend one or more scheduled clinic visits in the last 3 months, (d) commencing ART in the past 3 months, (e) who are pregnant and (f) with other psychosocial challenges or protection issues. Adolescents in need of enhanced support receive bi-weekly home visits, plus weekly phone calls and daily SMS. During the home visit, the CATS conduct an adherence assessment and provide adherence counseling as appropriate. A community health nurse accompanies the CATS where possible. If adolescents require more than one home visit every 2 weeks (for child protection cases, depression, ill-health), these are conducted by CATS with the caregiver present

Adolescents are allocated to the appropriate intervention level at enrollment, and re-assessed at 3-monthly intervals by the CATS in conjunction with the clinic nurses. Adolescents are moved between levels of intervention support as indicated by clinic attendance and other clinical factors as outlined in Table 1.

All participants at Zvandiri intervention sites are invited to attend a monthly support group, facilitated by a support-group leader (a volunteer nurse, teacher or social worker) in conjunction with the CATS, with supervision from the intervention coordinator. A standardized curriculum is used, focusing on improving health and treatment literacy, HIV disclosure, resilience and coping strategies, sexual and reproductive health, social networks and awareness of, and linkages to, services as required. Adolescents are actively followed up by the 
Table 1 Intervention components and levels of intervention support

\begin{tabular}{|c|c|c|}
\hline Level of care & Standard Zvandiri support & Enhanced Zvandiri support \\
\hline Eligibility criteria & $\begin{array}{l}\text { - A viral load }<1000 \text { copies/ml in last } 6 \text { months } \\
\text { - CD4 count } \geq 200 \text { cells/ml in last } 6 \text { months } \\
\text { - Attending all scheduled clinic visits in last } 3 \text { months } \\
\text { - Psychologically stable } \\
\text { - Safe }\end{array}$ & $\begin{array}{l}\text { - Commencing ART in past } 3 \text { months } \\
\text { - A viral load } \geq 1000 \text { copies/ml in the last } 6 \text { months } \\
\text { - CD4 count }<200 \text { cells/ml last } 6 \text { months } \\
\text { - Pregnancy } \\
\text { - Failing to attend } \geq 1 \text { scheduled clinic visit(s) in last } 3 \text { months } \\
\text { - Psychological distress } \\
\text { - Abuse or neglect }\end{array}$ \\
\hline Intervention & $\begin{array}{l}\text { - Monthly support group } \\
\text { - Monthly home visit } \\
\text { - Weekly SMS reminder } \\
\text { - Zvandiri Centre contact } \\
\text { - Caregiver workshop }\end{array}$ & $\begin{array}{l}\text { - Monthly support group } \\
\text { - Bi-weekly home visits } \\
\text { - Daily SMS reminder } \\
\text { - Weekly phone calls } \\
\text { - Zvandiri Centre contact } \\
\text { - Caregiver workshop } \\
\text { - Referral and linkages } \\
\text { - Community outreach visits }\end{array}$ \\
\hline
\end{tabular}

ART antiretroviral therapy, SMS short message service

CATS to maximize attendance at this support group. Adolescents identified to be at risk of harm are immediately referred to the Zvandiri intervention coordinator or clinic nurse for mental health services and/or management with the Department of Social Services.

\section{Zvandiri intervention conceptual framework}

The Zvandiri intervention is informed by the Unified Theory of Behavior (UTB) [22, 23]. The UTB is a validated conceptual framework that has previously been adapted to support adolescent behavior change within the context of a family centered approach. The UTB conceptualizes behavior in terms of two dimensions the immediate determinants of behavior, and of behavioral intention. It states that adherence to ART (for example) is more likely to occur if both (1) the determinants of adhering to ART and (2) the intention to adhere to ART are "aligned in favor of its enactment"
$[22,23]$. The five immediate determinants of behavior (i.e., ART adherence) are shown in Fig. 2, as are the immediate determinants of behavioral intention (i.e., intention to adhere to ART). We used this framework, combined with evidence from the academic literature about the determinants of adolescent HIV adherence and data from our formative work, to develop a modular Zvandiri intervention ensuring that each component of the framework is adequately addressed.

Caregivers of adolescents in the intervention arm are invited to a 12-session caregiver support group, facilitated by the intervention coordinator and CATS over 12 months. Sessions are in Shona, the participants' language. The sessions focus on improving caregivers' knowledge, skills and confidence of HIV and treatment literacy, communication and parenting and available support services. Two sessions are held jointly with adolescents.

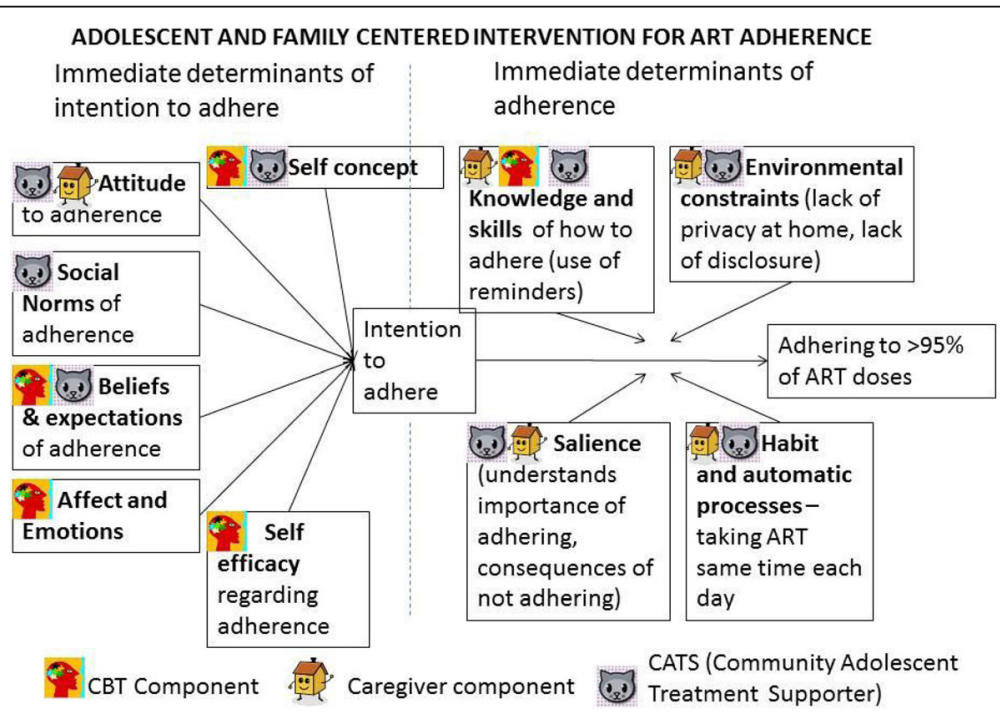

Fig. 2 Adolescent and family centered intervention for antiretroviral therapy (ART) adherence 


\section{Definition of endline}

The primary endpoint will be at 96 weeks post enrollment. There will be an 8-week window period on either side of this endpoint to allow flexibility in timing of visit (i.e., 88-104 weeks).

\section{Outcomes}

The primary outcome is the proportion of participants who have died or who have a viral load $\geq 1000$ copies $/ \mathrm{ml}$ at 96 weeks, assessed on a dried blood-spot sample. Secondary outcomes are assessed at 48 and 96 weeks, and are the proportion of participants:

1. Who are not retained in clinic services stratified according to the WHO definition [24]. Full retention is defined as attending $>80 \%$ of scheduled clinic appointments; partial retention as attending 30-80\% of scheduled appointments and non-retention as < 30\% attendance at scheduled appointments) [24]. Retention in services will be measured through attendance at scheduled clinic visits (scheduled visit defined as visit within \pm 7 days of exact date)

2. Who discontinue ART defined as completely stopping taking drugs for at least 3 months, as documented in clinic records (3 months is the minimum period associated with risk of progression to new clinical AIDS events or death) $[25,26]$

3. With depression, defined as scoring $\geq 10 / 27$ on the Patient Health Questionnaire (PHQ-9), a scale that has been widely used in Africa [27, 28] and recently validated for use in Zimbabwe [29]

4. With CMDs (depression and/or anxiety), defined as scoring $\geq 8 / 14$ on the Shona Symptom Questionnaire (SSQ-14) which was developed and validated in Zimbabwe using exemplary crosscultural methods [30]. This cut-point may be amended prior to locking the analysis plan, based on results of a separate validation study. Only two of its items relate to somatic symptoms making it useful in participants with physical disease. Severity of CMDs will also be assessed using the SSQ-14 as a continuous score

5. With poor quality of life, measured using a previously-validated Shona version of the European Quality of Life-5 Dimensions (EQ-5D) scale [31, 32]

Exploratory outcomes are assessed at 48 and 96 weeks, and are the proportion of participants:

1. Self-reporting disclosure of HIV status to sexual partners, assessed using a self-completed questionnaire

2. Self-reporting perceived stigma measured using the HIV/AIDS Stigma Instrument for people living with
AIDS (PLWA) (HASI-P) which has been validated in Africa [33]

\section{Sample size and potential power of the trial}

The total sample size of 500 participants recruited from 16 clusters will provide $80 \%$ power to detect a difference in detectable viral load of $35 \%$ among participants in the standard-care arm versus $18 \%$ in the intervention arm assuming $20 \%$ loss to follow-up and a coefficient of variation $(k)$ between clusters of 0.25 [34]. For secondary outcomes, the trial will have $90 \%$ power to detect, for example, a difference in non-complete attendance of $26 \%$ in the standard-care arm versus $10 \%$ in the intervention arm, and $80 \%$ power to detect a difference in mean SSQ-14 [30] score of 7.4 in the control arm and 4.8 in the intervention arm (standard deviation $(\mathrm{SD})=$ 3.74).

\section{Study schedule}

Figure 3 shows the study schedule which follows the Standard Protocol Items: Recommendations for Intervention Trials (SPIRIT) guidelines.

\section{Randomization}

Imbalance between arms was minimized by using restricted randomization and matching of clusters on size and number of people in HIV care. Sixteen clusters were allocated 1:1 to either the immediate intervention or the control arm. To maximize transparency and buy-in from key stakeholders, a public randomization procedure was undertaken on 5 July 2016 involving MoHCC, Community Advisory Board members, and district-level governance and medical representatives.

\section{Recruitment and enrollment procedures}

From the 16 clusters, a list of eligible participants was generated from the "pre-ART and ART Registers." At each cluster, selected village healthcare workers (VHCWs) were asked to mobilize potential participants after receiving training on trial objectives and procedures, as well as confidentiality aspects. The research team then held a trial-orientation meeting with the potential participants plus their caregivers. After orientation and screening, participants and caregivers who opted to take part in the trial and provided written consent, plus assent to enroll, were booked for enrollment procedures and baseline assessments. Recruitment and enrollment in the intervention and control arms occurred concurrently. At enrollment, participants were informed that they would be allocated to either the intervention or the control arm and told which one they were allocated to afterwards. Recruitment started on 15 August 2016 and concluded on 31 March 2017. 


\begin{tabular}{|c|c|c|c|c|c|}
\hline \multicolumn{6}{|c|}{ STUDY PERIOD } \\
\hline & \multirow{3}{*}{$\begin{array}{c}\text { Allocation } \\
0\end{array}$} & \multirow{3}{*}{$\begin{array}{l}\text { Enrolment } \\
\text { Baseline }\end{array}$} & \multicolumn{2}{|c|}{ Post-allocation } & \multirow{3}{*}{$\begin{array}{l}\text { Close-out } \\
104 \text { weeks }\end{array}$} \\
\hline & & & \multicolumn{2}{|c|}{ Interventions } & \\
\hline Time point & & & $\begin{array}{l}48 \text { weeks } \\
\text { follow-up }\end{array}$ & $\begin{array}{l}96 \text { weeks } \\
\text { follow-up }\end{array}$ & \\
\hline ALLOCATION: & $\mathbf{X}$ & & & & \\
\hline ENROLMENT: & & $\mathbf{X}$ & & & \\
\hline Eligibility screen & & $\mathrm{X}$ & & & \\
\hline Informed consent & & $\mathrm{X}$ & $\mathrm{X}$ & $\mathrm{X}$ & \\
\hline Survey procedures & & $\mathrm{x}$ & $\mathrm{x}$ & $\mathrm{x}$ & \\
\hline \multicolumn{6}{|l|}{ INTERVENTIONS: } \\
\hline \multicolumn{6}{|l|}{ Usual/standard care } \\
\hline \multicolumn{6}{|l|}{$\begin{array}{l}\text { Standard support \& caregiver } \\
\text { intervention }\end{array}$} \\
\hline \multicolumn{6}{|l|}{$\begin{array}{c}\text { Enhanced support \& caregiver } \\
\text { intervention }\end{array}$} \\
\hline \multicolumn{6}{|l|}{ ASSESSMENTS: } \\
\hline \multicolumn{6}{|l|}{ Primary outcome } \\
\hline Virological failure & & & $\mathrm{X}$ & $\mathrm{X}$ & \\
\hline \multicolumn{6}{|l|}{ Secondary outcomes } \\
\hline Retention in care & & & $\mathrm{X}$ & $\mathrm{x}$ & \\
\hline ART discontinuance & & & $\mathrm{x}$ & $\mathrm{x}$ & \\
\hline Depression & & & $\mathrm{X}$ & $\mathrm{X}$ & \\
\hline Common mental disorders & & & $\mathrm{X}$ & $\mathrm{X}$ & \\
\hline Quality of life & & & $\mathrm{X}$ & $\mathrm{X}$ & \\
\hline \multicolumn{6}{|l|}{ Exploratory outcomes } \\
\hline $\begin{array}{l}\text { Self-reported disclosure of HIV } \\
\text { status to sexual partners }\end{array}$ & & & $x$ & $x$ & \\
\hline Self-reported stigma & & & $\mathrm{x}$ & $\mathrm{x}$ & \\
\hline
\end{tabular}

Fig. 3 Schedule of enrollment, interventions and assessments following the Standard Protocol Items: Recommendations for Intervention Trials (SPIRIT) guidelines

\section{Quantitative data collection and management}

At enrollment, participants complete a questionnaire using an interviewer-administered, computer-assisted personal interview (CAPI) with more sensitive questions likely subject to socially desirability bias self-administered using an audiocomputer-assisted survey instrument (ACASI) [35, 36]. Questionnaire domains include: sociodemographic, socioeconomic, medical history, including history of opportunistic infections and hospitalization, HIV-testing history, ART history, information on clinic attendances, information on adherence (self-report), and psychological wellbeing. On completion of the questionnaire, all participants are asked to provide a finger-prick dried blood-spot sample for HIV viral- load testing and undergo a clinical exam to assess WHO stage. All viral-load test results will be returned to the clinic within 3 months to guide clinical care (in both the intervention and comparison arms). All trial participants are assessed by the research team, in addition to their routine clinic visits, after 48 and 96 weeks. At the follow-up visit, they will complete a shortened version of the questionnaire, have a finger-prick blood-spot sample taken for viral-load testing and have a physical examination to allow clinical staging.

\section{Quantitative data analysis}

Data collected using ACASI and CAPI is downloaded into a password-protected Access database. The data will 
be analyzed using Stata 14. Statistical analyses will be conducted and reported in line with Consolidated Standards of Reporting Trials (CONSORT) guidelines [37] including a flowchart. Baseline characteristics of enrolled participants and the number continuing through the trial, actively withdrawing, and passively lost to followup will be shown by arm. The outcome measures will be summarized at baseline, 48 and 96 weeks by intervention arm, using proportions or means (SD) as appropriate.

Analyses will be intention-to-treat. Analyses will be based on cluster-level summary measures, as individuallevel regression methods do not perform robustly when there are relatively few clusters per arm, especially for stratified cluster randomized trials [38]. For binary outcomes, the impact will be estimated by the risk ratio $(\mathrm{RR})$ and risk difference (RD). The stratum-specific risk ratios will be estimated as the ratio of the geometric mean risks between arms for each of the two strata, and the overall RR will be estimated as the weighted-average of these stratum-specific risk ratios. An approximate variance for the log of the mean risk in each arm will be estimated from the residual mean square from a twoway analysis of variance of community log-risk on strata and study arm. A 95\% confidence interval (CI) for the $R R$ will be estimated from this variance using a stratified $t$ test with 14 degrees of freedom. Similarly, a 95\% CI for the RD will be estimated from an analysis of variance of the mean risk on strata and study arm. For continuous outcomes, the measure of effect will be the mean difference between arms, analyzed in an analogous method based on mean scores in each facility.

Secondary planned analyses will examine the impact of the intervention separately by age group, gender, duration on treatment, with assessment of effectmodification of the intervention effect by facility type using a previously described method [39]. Pre-defined sensitivity analyses will include adjustment for baseline viral load and other variables imbalanced at baseline. We will conduct sensitivity analyses to investigate the effect of missing data by using multiple imputation, analyzed using an individual-level Poisson regression model, allowing for within-cluster correlation using generalized estimating equations.

\section{Qualitative data collection and management}

Twenty-six participants (20 adolescents - 10 male; 10 female - from across the age range plus six carers) from two intervention and two control communities will be purposively selected for three serial, in-depth interviews to understand the experiences of clinical care for children and young people living with HIV. In addition, four focus group discussions will be conducted with purposively selected participants and healthcare workers in both the enhanced intervention- and standard-care arms. All discussions will be audio-recorded; handwritten notes will be taken down as back-up.

\section{Qualitative data analysis}

Audio-recordings will be transcribed, translated into English, and coded by two independent coders in NVivo (QSR International, Melbourne, VIC, Australia). Coders will discuss discrepancies until consensus is reached. Codes will be grouped and emerging themes will then be identified. Analytic memos will be written for each theme. During write-up, themes and sub-themes will be illustrated with verbatim quotes.

\section{Cost-effectiveness data collection and analysis}

A cost-effectiveness analysis will be conducted using standardized survey instruments and taking a provider perspective, combining top-down and bottom-up costing. Study instruments will assemble data from program records and facility staff on relative costs of personnel, recurrent inputs and services, and capital costs (equipment, vehicles and training) which will be discounted at a 3\% rate. Any donated inputs will be valued at their opportunity costs determined using local market prices. Training and supervision will be valued according to opportunity cost of staff and CATS' time dedicated to these activities. Annual staff (providers and CATS) costs will be derived from staff roster information from program records and self-reports at the facility level; grades and salaries for each type of staff; and time and motion observation (the "gold standard" in measuring staff allocation of time through direct observation).

All cost data will be collected prospectively by month and analyzed in US dollars (the US\$ has been the principal currency in Zimbabwe since 2009). Any parameter uncertainty, such as influence of staff time per client, will be dealt with using univariate or multi-variate sensitivity analysis. As retention-in-care data (from trial) is only an intermediary measure of impact, we will use appropriate health-related quality of life (QALY) measures (e.g., EQ5D) to estimate QALYs gained for the purposes of costeffectiveness analysis. As intervention participants may transition between different states (standard Zvandiri support or enhanced Zvandiri support) over the course of the trial, a dynamic Markov model [40] will be used to track costs of care and utility at each point in time. A more realistic incremental cost-effectiveness ratio (ICER) threshold, WHO-CHOICE, which recommends a country gross domestic product (GDP)-dependent approach, will be adopted (http://www.who.int/choice).

\section{Process evaluation data collection and management}

The Intervention Monitoring and Process Evaluation framework provides a structure for data collection to capture progress along the hypothesized pathway from 
intervention activities to their intended outcomes. For the process evaluation, we hypothesize that:

If there are adequate inputs (human resources, partnerships and collaboration, established procedures and protocols, effective trainings)

These will ensure a smooth process of implementation, producing well-functioning outputs that will contribute to the achievement of the intervention's ultimate goals (improved physical and psychological health) (see Fig. 4)

\section{Program records}

Program data will be collected in intervention communities and regularly compiled to record uptake and attendance at community intervention, visits by CATS and use of clinical services.

\section{Process evaluation data analysis}

Service statistics and other quantitative data will be double entered into a password-protected Access database. Range and consistency checks will be performed. Quantitative data will be analyzed using Stata 14. Process evaluation qualitative data will be analyzed in the same manner as other qualitative data (already described).

\section{Discussion}

Effective strategies are urgently needed to reach the ambitious UNAIDS 90-90-90 target by 2020 (that $90 \%$ of all people living with HIV will know their HIV status, 90\% of all people with diagnosed HIV infection are receiving sustained ART, and $90 \%$ of all people receiving ART are virologically suppressed) [41]. To achieve this, high-

\begin{tabular}{|c|c|c|c|}
\hline \multicolumn{2}{|c|}{ Implementation of Intervention } & Coverage \& Intensity & Behaviour Change \\
\hline $\begin{array}{l}\quad \text { Inputs/ Preparation } \\
\text {-Partnership with relevant } \\
\text { partner agencies established } \\
\text { (MoHCC, MoPSLSW at } \\
\text { national, provincial and district } \\
\text { level) } \\
\text { - New staff recruited, trained and } \\
\text { deployed } \\
\text {-Clinic sites upgraded for } \\
\text { enhanced intervention } \\
\text {-Referral mechanisms put into } \\
\text { place } \\
\text {-Support group activity protocols } \\
\text { and tools } \\
\text {-Outreach activities to recruit } \\
\text { adolescents with HIV }\end{array}$ & $\begin{array}{c}\quad \text { Activities } \\
\text { - Allocation of CATS } \\
\text { - Support groups for } \\
\text { adolescents } \\
\text { - Caregivers support groups } \\
\text { - Supervision of CATS }\end{array}$ & 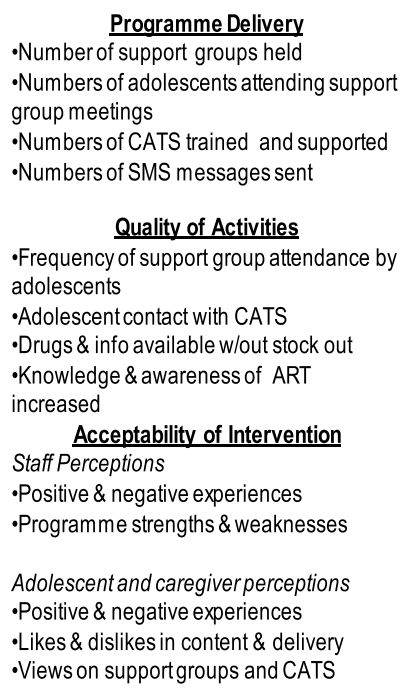 & $\begin{array}{l}\text { 1. Enabling Environment } \\
\text {-Strengthened supportive social } \\
\text { networks between adolescents } \\
\text {-Peer norms encouraging ART } \\
\text { adherence, disclosure to partners } \\
\text { 2. Sustained health behaviour } \\
\text {-Adherence to ARV } \\
\text { - Regular attendence at follow-up } \\
\text { clinical appointments } \\
\text {-Improved psychological health } \\
\text { - Increased disclosure with partners } \\
\text { - Increased condom use }\end{array}$ \\
\hline \multicolumn{4}{|c|}{ Tools } \\
\hline $\begin{array}{l}\text { - Checklist of planned activities, } \\
\text { with dates } \\
\text {-Staff records: \% of staff hired in } \\
2016 \text { still in place each year; } \\
\text { - Trainings:\# conducted and \% } \\
\text { staff attending } \\
\text {-Programme records: \# Support } \\
\text { group activities designed; } \\
\text {-\# sensitisation meetings to } \\
\text { inform stakeholders }\end{array}$ & $\begin{array}{l}\text {-Programme records } \\
\cdot ; \text { frequency of clinic openings; } \\
\text { average waiting time at clinic; } \\
\text {-CATS records: \# identified } \\
\text { and trained; \% retention of } \\
\text { CATS over time; CATS } \\
\text { supervision meetings held / } \\
\text { attended } \\
\text { •\# stakeholder meetings } \\
\text { attended }\end{array}$ & $\begin{array}{l}\text {-Questionnaire data: knowledge \& } \\
\text { awareness; reported adherence } \\
\text {-Programme records: analysis of clinic } \\
\text { records, stock-outs, CATS records } \\
\text {-Semi-structured interviews with HCWs } \\
\text { and CATS } 3 \text { times over project. } \\
\text {-In-depth interviews with adolescents } \\
\text { and caregivers } 3 \text { times over project at } \\
\text { intervention sites. } \\
\text { - Interviews with other stakeholders i.e. } \\
\text { referral organisations }\end{array}$ & $\begin{array}{l}\text {-Questionnaires: levels of social } \\
\text { support \& peer norms; \% adolescents } \\
\text { reporting condom use; adherence to } \\
\text { ARV; psychological distress; } \\
\text { disclosure to partners } \\
\text {-In-depth interviews with adolescents } \\
\text { in both intervention \& control sites: } \\
\text { Community stigma, social networks, } \\
\text { perception of norms and support } \\
\text {-Programme records: Clinic records on } \\
\text { attendance and prescription re-fills }\end{array}$ \\
\hline
\end{tabular}

Diminishing control- Project Diary to record external events affecting implementation

Fig. 4 Intervention Monitoring and Process Evaluation framework 
quality programs will need to ensure high levels of retention and adherence [11, 12].

Evidence-based strategies to support both adherence and retention in care of young people are required. This trial has the potential to generate robust evidence of the impact of the Zvandiri program as delivered in a realworld setting. If found to reduce rates of treatment failure, this would strengthen support for further scale-up across Zimbabwe and likely the region more widely. In addition, the results will directly influence policies and standard of care for HIV-infected children in Zimbabwe, regionally and internationally.

While the overall trial will answer the primary research question, "Does the Zvandiri program improve the physical and psychological health of adolescents living with HIV resulting in improved clinical outcomes and quality of life?" process evaluation and qualitative research will provide some understanding of why and how the intervention was able to lead to improved retention/ adherence along the HIV-care continuum (or suggest reasons for lack of effect). Additionally, process evaluation and qualitative research will suggest how the Zvandiri intervention could be modified for optimal impact during any further scale-up.

Finally, most countries including Zimbabwe have revised their HIV treatment guidelines in line with the WHO 2015 revised treatment guidelines which recommend a "test-and-start approach" for all those who are HIV infected, not just pregnant women [17]. Since a significant proportion of the likely beneficiaries of "test and start" are likely to be young people, this trial will help identify some of the challenges of this approach. Specifically, the trial will provide needed information on the capacity of primary care facilities to provide effective adherence support as well as support for the broader psychosocial issues that many of these young people face.

\section{Trial status}

At the time of initial manuscript submission recruitment for this trial was on-going but not yet complete.

\footnotetext{
Abbreviations

ACASI: Audio-computer-assisted survey instrument; AIDS: Acquired immunodeficiency syndrome; ART: Antiretroviral therapy; CAPI: Computerassisted personal interview; CATS: Community adolescent treatment supporters; CBT: Cognitive behavioral therapy; CD4: Cluster of differentiation 4; Cl: Confidence interval; CONSORT: Consolidated Standards of Reporting Trials; CRCT: Cluster randomized controlled trial; DSMB: Data Safety and Monitoring Board; EQ-5D: European Quality of Life-5 Dimensions; GDP: Gross domestic product; HASI-P: HIV/AIDS Stigma Instrument - PLWA; HIV: Human immunodeficiency virus; ICER: Incremental cost-effectiveness ratio; LMIC: Low- and middle-income countries; MoHCC: Ministry of Health and Child Care (Zimbabwe); PHQ: Patient Health Questionnaire; PLWA: People living with AIDS; QALY: Health-related quality of life; RD: Risk difference; RR: Risk ratio; SD: Standard deviation; SMS: Short message service; SPIRIT: Standard Protocol Items: Recommendations for Intervention Trials; SSQ: Shona Symptom Questionnaire; UNAIDS: Joint United Nations Program for HIV; USAID: United States Agency for International Development;
}

UTB: Unified Theory of Behavior; VHCW: Village healthcare worker; WHO: World Health Organization

\section{Acknowledgements \\ Study team would like to thank the adolescents, caregivers and health workers in the study districts for their participation in, and support of, the implementation of the trial.}

\section{Funding}

The study is funded by ViiV Healthcare's Positive Action for Adolescents Program (registered in England and Wales; No. 06990358). The funder had no role in study design, decision to publish, or preparation of the manuscript.

\section{Availability of data and materials}

Datasets will be deposited in publicly available repositories (where available and appropriate) in machine-readable format 3 years after the end of the study.

\section{Authors' contributions}

All authors contributed to the study design and writing of this paper. WM, NW, WMg, SB, RA, HAW and FMC are the study investigators and conceptualized and designed the study. JM is the study coordinator. CM is the trial health economist, and KM is a social scientist. TA provides technical support. All authors read and approved the final manuscript.

\section{Ethics approval and consent to participate}

The study has research ethics approval from the National Ethics Committee that oversees the ethical conduct of all research conducted in Zimbabwe Medical Research Council of Zimbabwe (MRCZ), reference number MRCZ/A/ 2032; University College London Research Ethics Committee, reference number 2538/004; and The London School of Hygiene and Tropical Medicine Observational/Interventions Research Ethics Committee, reference number 11042. All participants give written informed consent before enrolling in the study.

\section{Consent for publication}

Not applicable.

\section{Competing interests}

The authors declare that they have no competing interests.

\section{Publisher's Note}

Springer Nature remains neutral with regard to jurisdictional claims in published maps and institutional affiliations.

\section{Author details}

${ }^{1}$ Centre for Sexual Health and HIV/AIDS Research (CeSHHAR), 9 Monmouth Road, Avondale West, Harare, Zimbabwe. ${ }^{2}$ Department of International Public Health, Liverpool School of Tropical Medicine, Liverpool, UK. ${ }^{3}$ Africaid, Harare, Zimbabwe. ${ }^{4}$ School of Public Health, University of Sydney, Sydney, NSW, Australia. ${ }^{5}$ Department of Psychiatry, University of Zimbabwe College of Health Sciences, Harare, Zimbabwe. ${ }^{6}$ AIDS and TB Unit, Ministry of Health and Child Care, Harare, Zimbabwe. ${ }^{7}$ Health Services and Population Research Department, King's College London, London, UK. ${ }^{8}$ MRC Tropical Epidemiology Group, London School of Hygiene and Tropical Medicine, London, UK.

Received: 16 March 2017 Accepted: 18 September 2017 Published online: 20 October 2017

\section{References}

1. UNAIDS. 2014 HIV and AIDS estimates. Geneva: UNAIDS; 2015.

2. WHO. Health for the World's Adolescents: a second chance in the second decade. Geneva: WHO; 2014.

3. UNAIDS. UNAIDS report on the global AIDS epidemic 2013. Geneva: UNAIDS; 2013.

4. WHO. HIV and adolescents: guidance for HIV testing and counselling and care for adolescents living with HIV: recommendations for a public health approach and considerations for policy-makers and managers. Geneva: WHO; 2013 
5. Makadzange AT, et al. Clinical, virologic, immunologic outcomes and emerging HIV drug resistance patterns in children and adolescents in public ART care in Zimbabwe. PLoS One. 2015;10(12):e0144057.

6. Nachega JB, et al. Antiretroviral therapy adherence, virologic and immunologic outcomes in adolescents compared with adults in southern Africa. J Acquir Immune Defic Syndr. 2009;51(1):65-71.

7. Bygrave $\mathrm{H}$, et al. Antiretroviral therapy outcomes among adolescents and youth in rural Zimbabwe. PLoS One. 2012;7(12):e52856.

8. Taddeo D, Egedy M, Frappier JY. Adherence to treatment in adolescents. Paediatr Child Health. 2008;13(1):19-24.

9. WHO. Consolidated guidelines on the use of antiretroviral drugs for treating and preventing HIV infection: recommendations for a public health approach. Geneva: WHO; 2013.

10. MOHCC. Guidelines for Antiretroviral Therapy for the Prevention and Treatment of HIV in Zimbabwe. Harare: Ministry of Health and Child Care; 2013.

11. Bangsberg DR, et al. Adherence to protease inhibitors, HIV-1 viral load, and development of drug resistance in an indigent population. AIDS. 2000;14(4):357-66

12. Paterson $\mathrm{DL}$, et al. Adherence to protease inhibitor therapy and outcomes in patients with HIV infection. Ann Intern Med. 2000;133(1):21-30.

13. Mullen J, et al. Antiretroviral drug resistance among HIV-1 infected children failing treatment. J Med Virol. 2002;68(3):299-304.

14. San-Andres FJ, et al. Incidence of acquired immunodeficiency syndromeassociated opportunistic diseases and the effect of treatment on a cohort of 1115 patients infected with human immunodeficiency virus, 1989-1997. Clin Infect Dis. 2003;36(9):1177-85.

15. Van Dyke RB, et al. Reported adherence as a determinant of response to highly active antiretroviral therapy in children who have human immunodeficiency virus infection. Pediatrics. 2002;109(4):e61.

16. Vreeman $\mathrm{RC}$, et al. A systematic review of pediatric adherence to antiretroviral therapy in low- and middle-income countries. Pediatr Infect Dis J. 2008;27(8):686-91.

17. WHO. Guideline on when to start antiretroviral therapy and on preexposure prophylaxis for HIV. Geneva: WHO; 2015.

18. Mavhu W, et al. Enhancing psychosocial support for HIV positive adolescents in Harare, Zimbabwe. PLoS One. 2013;8(7):e70254.

19. Melissa S, Fullem A. Transitioning of care and other services for adolescents living with HIV in Sub-Saharan Africa. Arlington: USAID; 2012.

20. MOHCC. Guidelines for Antiretroviral Therapy for the Prevention and Treatment of HIV in Zimbabwe. Harare: Ministry of Health and Child Care; 2016.

21. Bygrave $H$, et al. Scaling up routine viral load monitoring in resource limited settings: field experience from antiretroviral therapy (ART) programmes in rural Zimbabwe and Malawi. 17th International Conference on AIDS and STls in Africa, Cape Town; 2013. abstract ADS037.

22. Jaccard J, Litardo HA, Wan CK. Subjective culture and social behavior. In: Adamopoulos J, Kashima Y, editors. Social psychology and cultural context. Thousand Oaks: Sage Publications; 1999. p. 95-106.

23. Jaccard J, Dodge T, Dittus P. Parent-adolescent communication about sex and birth control: a conceptual framework. New Dir Child Adolesc Dev. 2002;97:9-41.

24. WHO. Retention in HIV programmes: defining the challenges and identifying solutions: meeting report, 13-15 September 2011. Geneva: WHO; 2012.

25. Holkmann Olsen C, et al. Interruption of combination antiretroviral therapy and risk of clinical disease progression to AIDS or death. HIV Med. 2007;8(2): 96-104.

26. Kaufmann GR, et al. Interruptions of CART limits CD4 T-cell recovery and increases the risk for opportunistic complications and death. AIDS. 2011 ; 25(4):441-51.

27. Kroenke K, Spitzer RL, Williams JB. The PHQ-9: validity of a brief depression severity measure. J Gen Intern Med. 2001;16(9):606-13.

28. Monahan PO, et al. Validity/reliability of PHQ-9 and PHQ-2 depression scales among adults living with HIV/AIDS in western Kenya. J Gen Intern Med. 2009;24(2):189-97.

29. Chibanda D, et al. Validation of screening tools for depression and anxiety disorders in a primary care population with high HIV prevalence in Zimbabwe. J Affect Disord. 2016;198:50-5.

30. Patel V, et al. The Shona Symptom Questionnaire: the development of an indigenous measure of common mental disorders in Harare. Acta Psychiatr Scand. 1997;95(6):469-75.

31. Jelsma J, et al. The reliability of the Shona version of the EQ-5D. Cent Afr J Med. 2001;47(1):8-13.
32. Jelsma J, et al. The Validity of the Shona Version of the EQ-5D Quality of Life Measure. South Afr J Physiother. 2002;58(3):8-12.

33. Holzemer WL, et al. Validation of the HIV/AIDS Stigma Instrument - PLWA (HASI-P). AIDS Care. 2007:19(8):1002-12.

34. Hayes RJ, Moulton LH. Cluster randomised trials. Boca Raton: CRC Press; 2009.

35. Langhaug LF, et al. How you ask really matters: randomised comparison of four sexual behaviour questionnaire delivery modes in Zimbabwean youth. Sex Transm Infect. 2011;87(2):165-73.

36. Langhaug LF, Sherr L, Cowan FM. How to improve the validity of sexual behaviour reporting: systematic review of questionnaire delivery modes in developing countries. Trop Med Int Health. 2010;15(3):362-81.

37. Altman DG, et al. The revised CONSORT statement for reporting randomized trials: explanation and elaboration. Ann Intern Med. 2001;134(8):663-94.

38. Hayes R, Moulton L. Cluster randomised trials. Boca Raton: Taylor \& Francis; 2009.

39. Cheung YB, et al. A simple approach to test for interaction between intervention and an individual-level variable in community randomized trials. Trop Med Int Health. 2008;13(2):247-55.

40. Drummond MF, et al. Methods for the economic evaluation of health care programmes. Oxford: OUP; 2005.

41. UNAIDS. 90-90-90 An ambitious treatment target to help end the AIDS epidemic. Geneva: UNAIDS; 2014

\section{Submit your next manuscript to BioMed Central and we will help you at every step:}

- We accept pre-submission inquiries

- Our selector tool helps you to find the most relevant journal

- We provide round the clock customer support

- Convenient online submission

- Thorough peer review

- Inclusion in PubMed and all major indexing services

- Maximum visibility for your research

Submit your manuscript at www.biomedcentral.com/submit
) Biomed Central 